SUPERNOVAE \& SUPERSOFT X-RAY SOURCES 


\title{
ACCRETING WHITE DWARFS AND TYPE Ia SUPERNOVAE
}

\author{
MARIO LIVIO ${ }^{1}$, DAVID BRANCH ${ }^{2}$, L. R. YUNGELSON ${ }^{3}$ \\ FRANCESCA BOFFI ${ }^{1}$, E. BARON ${ }^{2}$ \\ 1. Space Telescope Science Institute, 3700 San Martin Drive, \\ Baltimore, MD 21218, USA \\ 2. Dept. of Physics and Astronomy, University of Oklahoma, \\ Norman, OK 73019, USA \\ 3. Institute of Astronomy of the Russian Acadamy of Sciences, \\ 48 Pyatnitskaya Str., 109017 Moscow, Russia
}

\begin{abstract}
The question of the possible progenitors of supernovae Type Ia ( $\mathrm{SNe} \mathrm{Ia}$ ) is examined. It is argued that $\mathrm{SNe}$ Ia are thermonuclear explosions of accreting $\mathrm{C}-\mathrm{O}$ white dwarfs. The existing observational evidence favors somewhat models in which the exploding star ignites carbon upon reaching the Chandrasekhar mass. A careful examination of all the potential progenitor classes reveals that when realization frequencies are combined with a variety of observational charcteristics, no single class emerges as containing the obvious progenitors. It is argued that coalescing white dwarfs or supersoft X-ray sources are the most likely progenitor systems. A few critical observations which could help identify the progenitors unambiguously are discussed.
\end{abstract}

\section{Characteristics and the basic model}

The defining characteristics in the spectra of supernovae Type Ia (SNe Ia) are the lack of lines of hydrogen and the presence of a strong red $\mathrm{Si}$ II absorption feature ( $\lambda 6355$ shifted to $\sim 6100 \AA$ ). The following are some of the observational characteristics of the class:

(i) nearly $90 \%$ of all $\mathrm{SNe}$ Ia form a homogeneous class in terms of their spectra, light curves and peak absolute magnitudes. The latter are given by $M_{\mathrm{B}} \simeq M_{\mathrm{V}} \simeq-19.75+5 \log \left(H_{0} / 50 \mathrm{~km} \mathrm{~s}^{-1} \mathrm{Mpc}^{-1}\right)$, with a dispersion of $\sigma\left(M_{\mathrm{B}}\right) \sim \sigma\left(M_{\mathrm{V}}\right) \sim 0.2$ (e.g. Tammannn \& Sandage 1995);

407

A. Evans and J. H. Wood (eds.), Cataclysmic Variables and Related Objects, 407-415. (C) 1996 Kluwer Academic Publishers. Printed in the Netherlands. 
(ii) near maximum light, the spectra are characterized by high velocity $\left(8000 \ldots 30000 \mathrm{~km} \mathrm{~s}^{-1}\right)$ intermediate mass elements $(\mathrm{O}-\mathrm{Ca})$. In the late, nebular phase, the spectra are dominated by forbidden lines of iron (e.g. Kirshner et al. 1993; Wheeler et al. 1995; Ruiz-Lapuente et al. 1995a);

(iii) in terms of explosion strength, SNe Ia can be roughly ordered as: SN 1991bg and SN 1992k represent the weakest events, followed by weak events like SN $1986 \mathrm{~g}$, followed by about $90 \%$ of all SNe Ia which are called 'normals', to the stronger-than-normal events like SN 1991t;

The luminosity function of SNe Ia declines very steeply on the bright side (e.g. Vaughan et al. 1995). Since selection effects cannot prevent the discovery of SNe which are brighter than the 'normals', this means that the normal SNe Ia are essentially the brightest;

(iv) fairly young populations appear to be most efficient at producing SNe Ia (they tend to be associated with spiral arms in spirals; Della Valle \& Livio 1994; Bartunov, Tsvetkov \& Filimonova 1994), but an old population $\left(\tau \gtrsim 410^{9} \mathrm{yr}\right)$ can also produce them (SNe Ia occur in ellipticals; Turatto, Cappellaro \& Benetti 1994). The explosion strength appears to be inversely correlated with the age of the stellar population (Ruiz-Lapuente, Burkert \& Canal 1995b).

As a consequence of the above points, and in particular point (iv), which shows that $\mathrm{SNe}$ Ia cannot be produced by core collapse of massive stars, the currently accepted model for SNe Ia is that they represent thermonuclear disruptions of mass accreting white dwarfs (WDs). Thus, the basic ingredient of the model for SNe Ia is the same type of object that is present in cataclysmic variables (CVs). The question that needs to be answered is: which binary system or systems are the immediate progenitors of SNe Ia? Before we address this question, we would like to explain the importance of identifying the progenitors.

\section{Why is the identification of the progenitors of SNe Ia important?}

Identifying the progenitor systems of SNe Ia is important for the following main reasons:

(i) in the absence of agreed upon detailed models for the explosion itself, a knowledge of the initial conditions and of the distribution of material in the vicinity of the exploding star are essential for the understanding of the explosion;

(ii) an identification of the progenitors will help (through the knowledge of the SNe Ia rate) to put constraints on models for binary evolution. In particular, it may help constrain the value of the common envelope effi- 
ciency parameter $\alpha_{\mathrm{CE}}$ (see e.g. Iben \& Livio 1993, Livio 1995 for reviews), which is presently very poorly known;

(iii) galaxy evolution depends on the input from supernovae in terms of energy (radiative and kinetic) and nucleosynthetic products and on the evolution of the $\mathrm{SN}$ rate with time;

(iv) the use of $\mathrm{SNe}$ Ia to determine $H_{0}$ and $q_{0}$ requires a knowledge of the evolution of the rate and the luminosity function of SNe Ia with cosmic time. Both of these quantities depend on the nature of the progenitors.

\section{Constraints on the basic model}

The basic model (thermonuclear disruption of an accreting WD) for SNe Ia can be further constrained. In particular, a combination of observations and theoretical calculations can be used in attempts to determine the required composition of the accreting white dwarf and the location in the WD (e.g. center versus shell) and the fuel where ignition first occurs.

\subsection{THE COMPOSITION OF THE ACCRETING WD}

The accreting WD could be, in principle, of $\mathrm{He}, \mathrm{CO}$ or $\mathrm{ONeMg}$ composition. However, in the case of a $\mathrm{He}$ WD, the composition of the ejected matter would be primarily $\mathrm{He}$ and ${ }^{56} \mathrm{Ni}$, which is inconsistent with the observations (see Sect. 1). In the case of $\mathrm{ONeMg}$ WDs there is no direct observational argument which can exclude them as potential progenitors. Theoretical calculations show, however, that the frequency of events that can be expected from such accretors is not high enough to explain the observed (rather deduced) SNe Ia rate (e.g. Livio 1993). Furthermore, detailed calculations show that accreting $\mathrm{ONeMg}$ WDs tend more to collapse quietly to form neutron stars, rather than to explode as SNe Ia (e.g. Gutierrez et al. 1995).

Consequently, it appears most likely that the accreting WDs that produce SNe Ia are of a CO composition.

\subsection{WHERE IN THE WD AND IN WHICH FUEL DOES IGNITION TAKE PLACE?}

There are presently two classes of models being considered: one in which carbon ignites at the WD center, when the WD reaches the Chandrasekhar mass, and the second, in which the accreted layer of helium $\left(\lesssim 0.2 \mathrm{M}_{\odot}\right)$ on top of a CO WD, ignites off-center, typically at sub-Chandrasekhar masses. In the case of the carbon ignitors, numerical simulations have shown that the energetics, light curve and composition as a function of ejection velocity are all quite consistent with observations (e.g. Branch et al. 1985; Harkness 1991; Höflich, Khokhlov \& Wheeler 1995). The fact that these models 
always explode at the Chandrasekhar mass has always been regarded as a potential explanation for the homogeneity of SNe Ia. The main difficulty with this class of models is related to statistics. Namely, it is not clear whether WDs in sufficient numbers to explain the SNe Ia rate can reach the Chandrasekhar mass (hydrogen and helium flashes which lead to mass loss make this difficult). A second, related difficulty, lies in the fact that WDs that are initially more massive than $1.2 \mathrm{M}_{\odot}$ (and therefore more likely, perhaps, to reach the Chandrasekhar mass), tend quietly to collapse to form neutron stars rather than to explode (Nomoto \& Kondo 1991), although it is not clear how firm this result is.

The helium (sub-Chandrasekhar) ignitors may produce (in principle) three different outcomes, two of which are certainly not normal SNe Ia. In the case of the 'direct double detonation' (DDD) (namely, one detonation wave propagating outward through the helium and the second inward, through the CO), the whole WD is burned into ${ }^{56} \mathrm{Ni}$ and He (Nomoto 1982; Woosley, Weaver \& Taam 1980). This is inconsistent with the observation of high-velocity intermediate mass elements.

The 'single detonation' (SD) case (the helium detonates but the CO core does not), in which the core may survive as a WD or disintegrate at low velocities, certainly does not correspond to normal SNe Ia (Nomoto 1982; Woosley, Taam \& Weaver 1986).

The only sub-Chandrasekhar model which has some chance to produce an explosion resembling a SN Ia is the 'indirect double detonation' (IDD) model. In this scenario, one detonation propagates outward through the helium, while an inward moving pressure wave results in ignition at the center (through the compression of the core), followed by an outward moving detonation (Livne \& Glasner 1990; Woosley \& Weaver 1994; Livne \& Arnett 1995; Höflich \& Khokhlov 1995).

Models of this type have been shown to produce light curves which are in agreement with those observed in SNe Ia. The main problems currently still existing with this model are: (i) the highest velocity ejecta have the wrong composition ( ${ }^{56} \mathrm{Ni}$ and He; e.g. Livne \& Arnett 1995), (ii) the light curve rises somewhat faster than observed (due to the presence of ${ }^{56} \mathrm{Ni}$ ) and the early colors are inconsistent with observations (Höflich \& Khokhlov 1995), (iii) one might expect this model to produce a gradual decline on the bright side of the luminosity function, in contradiction to the observed sharp decline. The last point would have been a consequence of the range of WD masses that the IDD model allows (more massive WDs produce brighter $\mathrm{SNe}$ ).

As a consequence of the above discussion we feel that carbon ignitors (at the Chandrasekhar mass) are presently favored by the observations, but that the uncertainties that still exist in the calculations of the helium ignitor 
IDD models suggest that this model should still be considered.

\section{Candidate progenitor systems}

We now return to the question of which binary systems could be the immediate progenitors of SNe Ia. Branch et al. (1995) examined this question in detail using realization frequencies of $\mathrm{SNe}$ Ia obtained from a variety of binaries. The calculations were performed with a population synthesis code of the type used by Yungelson et al. (1994, 1995). Of the many uncertainties which are involved in these calculations two are by far the most important ones in terms of their effects on the final results: the efficiency parameter in common envelope evolution, $\alpha_{\mathrm{CE}}$, and the fraction $\eta$ of the accreted mass, which a WD is able to retain. The efficiency parameter $\alpha_{\mathrm{CE}}$ describes the fraction of the gravitational energy change due to spiralling-in inside the common envelope which is deposited into envelope ejection. Since other sources of energy (e.g. recombination energy) may be involved, $\alpha_{\mathrm{CE}}$ could in principle be even larger than 1 (see Iben \& Livio 1993; Livio 1995 for reviews). At present, possible values of $\alpha_{\mathrm{CE}}$ are not reliably constrained neither observationally nor theoretically. A similar uncertainty exists in relation to the accumulation ratio $\eta$. Different numerical codes result in rather different fractions of the accreted mass which remain atop the WD following shell flashes (e.g. Starrfield et al. 1992; Prialnik \& Shara 1995). Furthermore, the accumulation ratio in a combination of hydrogen and helium flashes is even less certain (e.g. Iben \& Tutukov 1994; Kato, Saio \& Hachisu 1989).

Acknowledging the fact that these (and indeed other) uncertainties exist, Branch et al. (1995) adopted the following conservative assumption. A class of objects is not considered a major contributor to the SNe Ia rate, if the realization frequencies for young $\left(\sim 10^{8} \mathrm{yr}\right)$ and old $\left(\lesssim 10^{10} \mathrm{yr}\right)$ populations respectively satisfy: $\nu<10^{-4} \mathrm{yr}^{-1}, \nu<10^{-5} \mathrm{yr}^{-1}$ (the deduced rate for the Galaxy is $\sim 410^{-3} \mathrm{yr}^{-1}$; van den Bergh \& McClure 1994). In Table 1 we give the realization frequencies for all the relevant candidate progenitors for carbon and helium ignitors. As can be seen from the table, if one insists on only one class of progenitors, then only WD mergers have a sufficiently high frequency among the carbon ignitors. No single class can in fact produce the necessary frequencies among the helium ignitors, although symbiotic systems come close.

We will therefore examine now in some detail these two classes of objects.

WD mergers have been originally suggested as SNe Ia progenitors by Iben \& Tutukov (1984) and Webbink (1984). Recent hydrodynamic calculations have shown that when the lighter of the two WDs fills its Roche 
TABLE 1. Candidate progenitor systems and their realization frequencies

\begin{tabular}{lcc}
\hline Type of System & $\begin{array}{c}\text { Realization Frequency }\left(\mathrm{yr}^{-1}\right) \\
\text { for Young }\left(\sim 10^{8} \mathrm{yr}\right) \\
\text { Population }\end{array}$ & $\begin{array}{c}\text { Realization Frequency }\left(\mathrm{yr}^{-1}\right) \\
\text { for Old }\left(\lesssim 10^{10} \mathrm{yr}\right) \\
\text { Population }\end{array}$ \\
\hline $\begin{array}{l}\text { a. Carbon Ignitors } \\
\text { WD accreting from Roche }\end{array}$ & $10^{-5}$ & - \\
lobe filling subgiant or \\
giant
\end{tabular}

lobe (the two WDs having been brought together by gravitational radiation), it is totally dissipated within a few orbital periods, to form a thick disk-type configuration (rotationally supported) around the primary (Rasio \& Shapiro 1995; Benz et al. 1990). The subsequent evolution of the system is not entirely clear, since it is not known if central or off-center ignition will occur (e.g. Mochkovitch \& Livio 1989, 1990; Mochkovitch et al. 1995). Two points which may give some confidence in the realization frequencies obtained for mergers are as follows. (i) The population synthesis calculations indicate that for populations younger than $\sim 10^{8.5} \mathrm{yr}$, the total (combined) mass of the merging WDs is of order $2.1 \mathrm{M}_{\odot}$, while it converges to the Chandrasekhar mass for older populations (Tutukov \& Yungelson 1995). This may be consistent with the inverse correlation found between 
explosion strength and population age (Ruiz-Lapuente et al. 1995b). (ii) All the recently discovered double WD systems (Marsh 1995; Marsh, Dhillon \& Duck 1995) fall into the peak of the period distribution predicted by the population synthesis calculations of Yungelson et al. (1994). On the other hand, it should be realized that some of the paths which lead to the formation of double WD systems involve two common envelope phases and thus they are necessarily uncertain (see e.g. Livio 1994).

It is important to note that what has been considered until recently as a fatal difficulty for the merger scenario, the tentative detection of hydrogen in the supernova $1990 \mathrm{~m}$ (Polcaro \& Viotti 1991), no longer poses a problem, since this detection has been shown to be erroneous (Della Valle, private communication). A difficulty that still exists is statistical in nature; among the nine double WD systems discovered so far (Saffer, Liebert \& Olszewski 1988; Bragaglia et al. 1990; Marsh et al. 1995; Marsh 1995) not a single one is a SN Ia progenitor candidate, either because the orbital period is too long for the two WDs to merge in a Hubble time, or because the total mass is significantly lower than the Chandrasekhar mass. Other searches for double WD systems have given rather negative results (e.g. Robinson \& Shafter 1987; Foss, Wade \& Green 1991). While Yungelson et al. (1994) have shown that the results of any single one of these searches are still not in conflict with the possibility of producing SNe Ia at a rate compatible with observations $\left(\sim 310^{-3} \mathrm{yr}^{-1}\right)$, the combined negative results of all the searches starts to place uncomfortable constraints on this scenario (see also Renzini 1995).

As noted above, if helium ignitors represent the correct model (which presently appears difficult to accept), then symbiotic systems come the closest to producing the required frequency of SNe Ia. A real observational test for this scenario can come from deep radio observations performed immediately after the explosion. Due to the high wind mass loss rates observed from the giants in symbiotic systems $\left(\dot{M}_{\mathrm{w}} \sim 10^{-6} \mathrm{M}_{\odot} \mathrm{yr}^{-1}\right.$, the circumstellar density in the vicinity of these systems should be high enough to produce detectable radio emission shortly after the SN explosion. Boffi \& Branch (1995) showed that for $\dot{M}_{\mathrm{w}} \sim 10^{-6} \mathrm{M}_{\odot} \mathrm{yr}^{-1}$, the peak of the emission should occur about a week after the explosion and the expected peak flux is about $30 \mathrm{mJy}$ for a distance of $4 \mathrm{Mpc}$. Thus, the upper limit to the radio flux from (the rather weak) SN 1986g (Eck et al. 1995), appears to indicate that the progenitor of this supernova at least, was probably not a symbiotic system. Finally, persistent supersoft X-ray sources, which involve a WD accreting at a rate $\gtrsim 10^{-7} \mathrm{M}_{\odot} \mathrm{yr}^{-1}$ from a subgiant companion (van den Heuvel et al. 1992; Rappaport, Di Stefano \& Smith 1994; Yungelson et al. 1995) and burning hydrogen stably, include (in principle) the necessary ingredients for a system to reach the Chandrasekhar mass. While 
the realization frequency for such events appears presently to be too low (Yungelson et al. 1995), it is possible that this merely represents the uncertainties involved in population synthesis calculations. Hence, the class of supersoft X-ray sources should be kept in mind as potentially viable progenitor candidates.

\section{General problems with current models}

In the previous sections we described difficulties that arise in relation to the identification of SNe Ia progenitors. Some of the problems involved are well exemplified by the fact that whichever the preferred model may be, some questions remain unanswered. For example, if we decide that the helium (sub-Chandrasekhar) ignitors are not the correct model for the majority of SNe Ia, this may mean that either the realization frequencies given in Table 1 are very wrong (which is quite possible, in view of the many uncertainties), or that off-center helium ignition leads to consequences that are very different from IDDs (e.g. single detonations, although current models do not favor them). In the latter case, however, it may mean that a large population of these subluminous (as far as SNe go) explosions awaits detection. Conversly, if we decide that the helium ignitors do represent the correct model for SNe Ia, then it is not clear why current models predict that, for WD masses larger than $\sim 0.9 \mathrm{M}_{\odot}$, a direct double detonation should be obtained. The absence of these more luminous events in the observational sample seems to suggest that direct double detonations never occur in nature, in contradiction to theoretical expectations.

Acknowledgement. This research has been supported in part by NASA Grant NAGW-2678 at the Space Telescope Science Institute.

\section{References}

Bartunov, O.S., Tsvetkov, D.Yu., Filimonova, I.V., 1994, PASP, 106, 1276

Benz, W., Bowers, R.L., Cameron, A.G.W., Press, W.H., 1990, Ap. J., 348, 647

Boffi, F., Branch, D., 1995, PASP, 107, 347

Bragaglia, A., Greggio, L., Renzini, A., D’Odorico, S., 1990, Ap. J., 365, L13

Branch, D., Doggett, J.B., Nomoto, K., Thielemann, F.-K., 1985, Ap. J., 294, 619

Branch, D., Livio, M., Yungelson, L.R., et al., 1995, PASP, in press

Della Valle, M., Livio, M., 1994, Ap. J., 423, L31

Eck, C., Cowan, J.J., Roberts, D.A., et al., 1995, Ap. J., submitted

Foss, D., Wade, R.A., Green, R.F., 1991, Ap. J., 374, 281

Gutierrez, J., Garcia-Berro, E., Iben, I., et al., 1995, preprint

Harkness, R.P., 1991, in "Supernovae", ed. S.E. Woosley (New York: Springer-Verlag), $\mathrm{p} 454$

Höflich, P., Khokhlov, A., 1995, Ap. J., in press

Höflich, P., Khokhlov, A., Wheeler, J.C., 1995, Ap. J., 444, 831

Iben, I. Jr., Livio, M., 1993, PASP, 105, 1373

Iben, I. Jr., Tutukov, A.V., 1984, Ap. J. Supp., 54, 335 
Iben, I. Jr., Tutukov, A.V., 1994, Ap. J., 431, 264

Kato, M., Saio, H., Hachisu, I., 1989, Ap. J., 340, 509

Kirshner, R.P., et al., 1993, Ap. J., 415, 589

Livio, M., 1993, in "Cataclysmic Variables and Related Physics", eds O. Regev, G. Shaviv, Ann. Israel Phys. Soc., 10, p57

Livio, M., 1994, Mem. S. A. It., 65, 49

Livio, M., 1995, in "Evolutionary Processes in Binary Stars", eds. R.A.M.J. Wijers et al., (Dordrecht: Kluwer), in press

Livne, E., Arnett, D., 1995, Ap. J., in press

Livne, E., Glasner, A., 1990, Ap. J., 361, 244

Marsh, T.R., 1995, MNRAS, in press

Marsh, T.R., Dhillon, V.S., Duck, S.R., 1995, MNRAS, in press

Mochkovitch, R., et al., 1995, in preparation

Mochkovitch, R., Livio, M., 1989, A\&A, 209, 111

Moch kovitch, R., Livio, M., 1990, A\&A, 236, 378

Nomoto, K., 1982, Ap. J., 257, 780

Nomoto, K., Kondo, Y., 1991, Ap. J., 367, L19

Polcaro, V.F., Viotti R., 1991, A\&A, 242, L9

Prialnik, D., Shara, M.M., 1995, AJ, 109, 1735

Rappaport, S., Di Stefano, R., Smith, J.D., 1994, Ap. J., 426, 692

Rasio, F.A., Shapiro, S., 1995, Ap. J., 438, 887

Renzini, A., 1995, in "Supernovae", ed. R. McCroy (Cambridge: Cambridge University Press), in press

Robinson, E.L., Shafter, A.W., 1987, Ap. J., 322, 296

Ruiz-Lapuente, P., Kirshner, R.P., Phillips, M.M., et al., 1995a, Ap. J., 439, 60

Ruiz-Lapuente, P., Burkert, A., Canal, R., 1995b, Ap. J., 447, L69

Saffer, R.A., Liebert, J., Olszewski, E., 1988, Ap. J., 334, 947

Starrfield, S., Shore, S.N., Sparks, W.M., et al., 1992, Ap. J., 391, L71

Tammannn, G.A., Sandage, A., 1995, Ap. J., in press

Tutukov, A.V., Yungelson, L.R., 1995, in "Cataclysmic Variables", eds A. Bianchini et al., Kluwer, p495

Turatto, M., Cappellaro, E., Benetti, S., 1994, AJ, 108, 202

van den Bergh, S., McClure, R.D., 1994, Ap. J., 425, 205

van den Heuvel, E.P.J., Bhattacharya, D., Nomoto, K., Rappaport, S.A., 1992, A\&A, 262, 97

Vaughan, T.E., Branch, D., Miller, D.L., Perlmutter, S., 1995, Ap. J., 439, 558

Webbink, R.F., 1984, Ap. J., 277, 355

Wheeler, J.C., Harkness, R.P., Khokhlov, A.V., Höflich, P., 1995, Phys. Rep., in press

Woosley, S.E., Taam, R.E., Weaver, T.A., 1986, Ap. J., 301, 601

Woosley, S.E., Weaver, T.A., 1994, Ap. J., 423, 371

Woosley, S.E., Weaver, T.A., Taam, R.E., 1980, in "Type Ia Supernovae", ed. J.C. Wheeler (Austin: University of Texas), p96

Yungelson, L.R., Livio, M., Tutukov, A.V., Saffer, R.A., 1994, Ap. J., 420, 336

Yungelson, L.R., Livio, M., Truran, J.W., et al., 1995, Ap. J., submitted 